\title{
EDITORIAL
}

\section{Community engagement}

\author{
Crowdsourcing efforts, such as those involved in the Escherichia coli O104 outbreak and, most \\ recently, ash dieback disease, reflect a new era of community engagement in genomic analyses.
}

Emerging infections are probably most often thought of as affecting humans, and consequently, human infections receive most attention in the mainstream media, the new coronavirus outbreak being a case in point. In recent years, devastating epidemics in other species, such as chytridiomycosis in frogs (caused by Batrachochytrium dendrobatidis) and white nose syndrome in bats (caused by Geomyces destructans), have pricked the collective consciousness. Livestock infections occasionally also warrant a mention, with the ongoing outbreak of Schmallenberg virus, which affects newborn ruminants, being the most recent example here in the United Kingdom.

However, less attention is typically paid to emerging (or indeed established) infections in plants, despite the fact that the economic impact of infectious diseases in plants such as food crops is enormous. Global food security is a topic that we will return to later in the year, as part of a special focus issue on plant-microorganism interactions. For now, we wish to highlight an emerging plant disease that, for once, has been relatively widely reported by the UK media. Ash dieback is caused by the fungus Chalara fraxinea (teleomorph Hymenoscyphus pseudoalbidus), with infection causing leaf loss and dieback of the crown of ash trees. Although it is believed that a chronic infection can be established, the disease can be lethal, particularly in young trees. First reported in Poland in the early 1990s, the disease spread, reaching Scandinavia and Germany in the early 2000s. Over the past decade, an estimated 95\% of ash trees in Denmark have been infected.

In the UK, signs of ash dieback were first reported in imported stock in a nursery in February 2012. According to the UK Forestry Commission, ash trees cover approximately 130,000 hectares of UK woodland, accounting for more than $10 \%$ of broad-leaved woodland by area. This does not include private woodland, but estimates in a recent Confederation of Forest Industries impact report suggest that almost $40 \%$ of broad-leaved seedlings and saplings in private woodland are ash. Despite movement restrictions and a ban on imports of ash seeds, plants and trees, the infection has spread rapidly in the UK, and as of the beginning of March 2013, an estimated 390 sites were affected, including 170 in natural environments such as established woodland.
At present, knowledge on the pathogen is scant. A group of researchers led by Dan MacLean and colleagues at The Sainsbury Laboratory in Norwich, UK, decided that swift action was required to obtain and analyse $C$. fraxinea genomic information. Having generated genome sequence data from a purified culture of $C$. fraxinea as well as transcriptomic data from infected trees, rather than analyse the data themselves and follow the traditional publication route, they released the raw data immediately and established a website and accompanying GitHub repository to allow crowdsourcing analysis. The use of GitHub allows users to download the raw data and then carry out whatever type of analysis they are interested in contributing, whether it be assembly, alignment or annotation. All contributors are listed and their specific contributions are noted to ensure a fair attribution of effort. To date, the analyses that have been carried out include gene predictions and analysis of the mating-type locus, and additional input will be very welcome ${ }^{1}$.

Of course, crowdsourcing has previously been used in microbial genomic analysis. In a well-publicized effort in 2011, crowdsourcing was first used in the analysis of an outbreak of Shiga toxin-producing Escherichia coli in Germany. The first sequence data were publicly released by BGI on 2 June 2011 under a Creative Commons 0 license. Within 24 hours, the first genome assembly was available, and within 7 days, multiple analyses had been carried out by bioinformaticians worldwide. These analyses provided rapid insights into the phylogenetic lineage and virulence of the pathogen along with, crucially, the information necessary to design diagnostic PCR primers ${ }^{2}$.

Community engagement in genomic analyses is not new. Many of the early whole-genome sequences of bacterial and fungal species were achieved by large collaborations involving sequencing centres and established investigators who were recognized authorities on the species in question and were thus able to persuade funding bodies to invest in this new technology and then drive the annotation effort. So the engagement may not be not new, but with such crowdsourcing efforts, the nature of the community has changed dramatically and for the better. We concur with the rallying cry from Dan

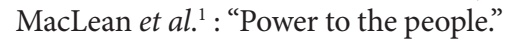

1. MacLean, D. et al. Gigascience 2, 2 (2013)

2. Rohde, H. et al. New Engl. J. Med. 365, 718-724 (2011) 